\title{
To thin or not to thin?
}

\author{
Kevin J. Koomalsingh, MD, and Nahush A. Mokadam, MD
}

\author{
From the Division of Cardiothoracic Surgery, University of Washington, Seattle, Wash. \\ Disclosures: N.A.M. is a consultant to Abbott and Medtronic and an investigator for Abbott, Medtronic, and Syn- \\ Cardia. K.J.K has nothing to disclose with regard to commercial support. \\ Received for publication Nov 6, 2017; accepted for publication Nov 8, 2017; available ahead of print Jan 2, 2018. \\ Address for reprints: Nahush A. Mokadam, MD, FACC, FACS, LeRoss Endowed Professor in Cardiovascular \\ Surgery, Division of Cardiothoracic Surgery, University of Washington, 1959 NE Pacific St, Box 356310, \\ Seattle, WA 98195 (E-mail: mokadamn@uw.edu). \\ J Thorac Cardiovasc Surg 2018;155:1007-8 \\ $0022-5223 / \$ 36.00$ \\ Copyright (C) 2017 by The American Association for Thoracic Surgery \\ https://doi.org/10.1016/j.jtcvs.2017.11.023
}

Surgical ablation for atrial fibrillation remains an area of active investigation and interest for cardiac surgeons. Improved technology, high rhythm conversion rates, longterm success, and a growing interest in minimally invasive and hybrid approaches have resurrected arrhythmia surgery. Still, we are plagued by the lack of an appropriate anticoagulation regimen after surgical ablation. Existing recommendations and guidelines are extrapolations from catheter-based techniques and do not account for the complexities in dealing with a postoperative cardiac surgical patient. ${ }^{1,2}$ In addition, they emphasize variables such as the $\mathrm{CHADS}_{2}$ (congestive heart failure, hypertension, age $\geq 75$ years, diabetes mellitus, stroke [double weight]) score, which have been insufficient at guiding oral anticoagulation therapy (OAC) therapy in our patient population. ${ }^{3}$ We have therefore witnessed an extreme variability in practice patterns.

In this month's issue of the Journal, Murashita and colleagues ${ }^{4}$ help elucidate some of the enigma surrounding postoperative anticoagulation after surgical ablation. In a 4-year follow up, they compared patients discharged with and without OAC. All patients had undergone a Cox maze IV surgical ablation, most performed as a concomitant procedure. Murashita and colleagues ${ }^{4}$ are to be commended for constructing a well-executed study. They were incredibly successful at rhythm conversion. In fact, nearly $98 \%$ of their patients were discharged in normal sinus rhythm, with $77 \%$ remaining in normal sinus rhythm at 4 years. The rate of the primary end point, cerebrovascular accident, was lower than $1 \%$ per patient-year in both cohorts. As expected, major bleeding events were more prevalent in the OAC group; however, the difference was not statistically significant. The findings as a whole are intriguing, and they bolster the argument for withholding OAC after surgical ablation for atrial fibrillation.

Nevertheless, the Achilles' heel of atrial fibrillation remains the inherent variability in surgical and medical management. A "maze" procedure can vary anywhere from a simple pulmonary vein isolation to a full biatrial lesion set, will likely continue.

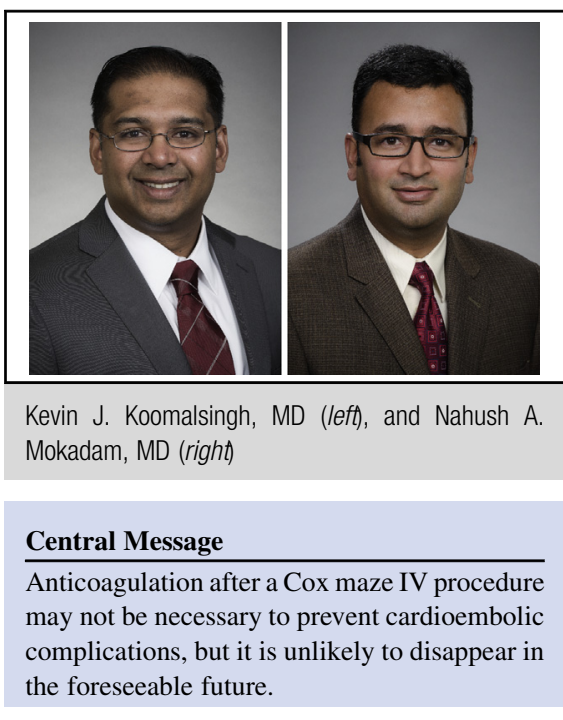

See Article page 997.

and even a "full" biatrial lesion set is variable from surgeon to surgeon and center to center. Furthermore, the left atrial appendage can be managed in a variety of ways, each with its corresponding failure rate. Medical management adds yet another layer of complexity. For example, a recent Canadian study uncovered not only inconsistent anticoagulation prescribing patterns by referring cardiologists but also significant deviations from existing guidelines and recommendations for long-term management of atrial fibrillation. ${ }^{5}$ The multidimensional scope of this problem is therefore likely to be underappreciated. Another wrench in the works stems from recent changes in anticoagulation guidelines after valvular heart surgery. ${ }^{2}$ Because the vast majority of Cox maze IV procedures are performed concomitantly, is it practical to withhold anticoagulation in just a mere handful of cases? Should more patients undergo a stand-alone Cox maze IV procedure? Would a randomized clinical trial help elucidate this issue?

Perhaps if all surgeons were able to generate a 9-month freedom from atrial fibrillation of $90 \%$, a left atrial appendage failure rate of $0 \%$, and a surgical ablation time of 16 minutes, unification and standardization would be within reach. We are certainly enthusiastic about the possibility of discontinuing anticoagulation in our patients undergoing surgical ablation. We, as a surgical society, need to recognize the tremendous opportunity in unifying this initiative. Until then, however, the warfarin prescriptions 


\section{References}

1. Calkins H, Kuck KH, Cappato R, Brugada J, Camm AJ, Chen SA, et al; Heart Rhythm Society Task Force on Catheter and Surgical Ablation of Atrial Fibrillation. 2012 HRS/EHRA/ECAS expert consensus statement on catheter and surgical ablation of atrial fibrillation: recommendations for patient selection, procedural techniques, patient management and follow-up, definitions, endpoints, and research trial design: a report of the Heart Rhythm Society (HRS) Task Force on Catheter and Surgical Ablation of Atrial Fibrillation. Developed in partnership with the European Heart Rhythm Association (EHRA), a registered branch of the European Society of Cardiology (ESC) and the European Cardiac Arrhythmia Society (ECAS); and in collaboration with the American College of Cardiology (ACC), American Heart Association (AHA), the Asia Pacific Heart Rhythm Society (APHRS), and the Society of Thoracic Surgeons (STS). Endorsed by the governing bodies of the American College of Cardiology Foundation, the American Heart Association, the European Cardiac Arrhythmia Society, the European Heart Rhythm Association, the Society of Thoracic Surgeons, the Asia Pacific Heart
Rhythm Society, and the Heart Rhythm Society. Heart Rhythm. 2012;9: 632-96.e21.

2. Nishimura RA, Otto CM, Bonow RO, Carabello BA, Erwin JP III, Fleisher LA, et al. 2017 AHA/ACC focused update of the 2014 AHA/ACC guideline for the management of patients with valvular heart disease: a report of the American College of Cardiology/American Heart Association Task Force on Clinical Practice Guidelines. Circulation. 2017;135:e1159-95.

3. Ad N, Henry L, Shuman DJ, Holmes SD. A more specific anticoagulation regimen is required for patients after the Cox-maze procedure. Ann Thorac Surg. 2014;98: 1331-8.

4. Murashita T, Rankin JS, Wei LM, Roberts HG, Alkhouli MA, Badhwar V. Oral anticoagulation may not be necessary for patients discharged in sinus rhythm following the Cox maze IV procedure. J Thorac Cardiovasc Surg. 2018;155:997-1006.

5. Chung J, Sami M, Albert C, Varennes B. Variations in anticoagulation practices following the maze procedure. J Atr Fibrillation. 2015;8:1273. 